\title{
Measurement of $\mathrm{R}$ in $2-5 \mathrm{GeV}$ with BES-II at BEPC
}

\section{Jiangchuan Chen* (Representing BES Collaboration)}

Div.1, IHEP of $C A S$

E-mail: 'chenjomaji

ABstRACT: We report values of $R=\sigma\left(e^{+} e^{-} \rightarrow\right.$ hadrons $) / \sigma\left(e^{+} e^{-} \rightarrow \mu^{+} \mu^{-}\right)$for 85 centerof-mass energies between 2 and $5 \mathrm{GeV}$ measured with the upgraded Beijing Spectrometer at the Beijing Electron-Positron Collider.

\section{Motivation}

$R$ is the lowest order cross section for $e^{+} e^{-} \rightarrow \gamma^{*} \rightarrow$ hadrons in units of the lowest-order QED cross section for $e^{+} e^{-} \rightarrow \mu^{+} \mu^{-}$, namely $R=\sigma\left(e^{+} e^{-} \rightarrow\right.$ hadrons $) / \sigma\left(e^{+} e^{-} \rightarrow \mu^{+} \mu^{-}\right)$, where $\sigma\left(e^{+} e^{-} \rightarrow \mu^{+} \mu^{-}\right)=\sigma_{\mu \mu}^{0}=4 \pi \alpha^{2}(0) / 3 s$. A precision measurement of $\mathbf{R}$ will reduce the uncertainty on $\alpha\left(M_{z}\right)$, which is one of the three basic SM parameters, and thus allow for more precise tests of the SM. The R measurement will narrow the predicated mass window of Higgs boson $m_{H}$, which depends critically on $\alpha\left(M_{Z}\right)$. And the new value of $\mathrm{R}$ will contribute to the interpretation of $a_{\mu}$ too, where $20 \%$ of theory uncertainty comes from $\mathrm{R}$ in $2-5 \mathrm{GeV}$ energy region.

Values of $R$ in the center-of-mass $(\mathrm{cm})$ energy range below $5 \mathrm{GeV}$ were measured previously about 20 years ago with a precision of $15-20 \%$ (see Table

\begin{tabular}{|c|c|c|c|}
\hline$E_{c m}(\mathrm{GeV})$ & $1-5$ & $5-10$ & $10-m_{Z}$ \\
\hline$\Delta R / R(\%)$ & $15-20$ & $5-10$ & $2-7$ \\
\hline
\end{tabular}

Table 1: Uncertainties of $R$ in different energy regions

\section{Apparatus}

The measurements were carried out with the upgraded Beijing Spectrometer (BESII) at the Beijing Electron-Positron Collider (BEPC). BESII is a conventional collider detector

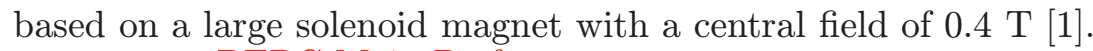

BEPC Main Performance

\begin{tabular}{|c|l|}
\hline & \multicolumn{1}{|c|}{ Performance } \\
\hline peak Luminosity: & $\begin{array}{l}5 \times 10^{30} \mathrm{~cm}^{-2} \mathrm{~s}^{-1} \\
\text { (at } \mathrm{J} / \psi \text { resonance) }\end{array}$ \\
\hline Energy spread: & $0.58 \mathrm{MeV}$ \\
\hline Bunch length: & $5 \mathrm{~cm}$ \\
\hline
\end{tabular}

BESII Main Performance

\begin{tabular}{|c|l|}
\hline Subdetector & Performance \\
\hline MDC & $\sigma_{p} / p \sim 1.8 \% \sqrt{1+p^{2}}$ \\
\hline TOF & $\sigma_{T O F} \sim 180 \mathrm{ps}$ \\
\hline BSC & $\sigma_{E} / E \sim 21 \% / \sqrt{E}$ \\
\hline
\end{tabular}

${ }^{*}$ Speaker. 


\section{Measurement of $\mathrm{R}$ at BESII}

Experimentally, the value of $R$ is determined from the number of observed hadronic events, $N_{\text {had }}^{\text {obs }}$, by the relation

$$
R=\frac{\sigma_{h a d}^{0}}{\sigma_{\mu \mu}^{0}}=\frac{N_{h a d}^{o b s}-N_{b g}-\sum_{l} N_{l l}-N_{\gamma \gamma}}{\sigma_{\mu \mu}^{0} \cdot \varepsilon_{h a d} \cdot \varepsilon_{t r g} \cdot(1+\delta) \cdot \mathcal{L}}
$$

where $N_{b g}$ is the number of beam-associated background events; $\sum_{l} N_{l l},(l=e, \mu, \tau)$ are the numbers of lepton-pair events from one-photon processes and $N_{\gamma \gamma}$ is the number of two-photon process events that are misidentified as hadronic events; $L$ is the integrated luminosity; $\delta$ is the effective initial state radiative (ISR) correction; $\epsilon_{\text {had }}$ is the detection efficiency for hadronic events; and $\epsilon_{t r g}$ is the trigger efficiency.

BES carried out 2 runs of $\mathbf{R}$ scan experiment.

The First Run (Spring '98): 6 energy points scanned, large sample @2.6 and 3.55 GeV to tune LUND parameters; Results was published in PRL [2].

The Second Run (Spring '99): 85 energy points, and 24 points also with separatedbeam data, 7 points also with single-beam data.

\section{Data Analysis}

We use a set of requirements on fiducial regions, vertex positions, track fit quality, maximum and minimum Barrel Shower Counter (BSC) energy deposition, track momenta and time-of-flight hits to preferentially distinguish one-photon multi-hadron production from all possible contamination mechanisms. After the imposition of these requirements, the remaining backgrounds are due to cosmic rays, lepton pair production, two-photon interactions and single-beam-related processes. Additional requirements are imposed on two-prong events, for which cosmic ray and lepton pair backgrounds are especially severe.

An acceptable charged track must be in the polar angle region $|\cos \theta|<0.84$, have a good helix fit, and not be clearly identified as an electron or muon. The distance of closest approach to the beam axis must be less than $2 \mathrm{~cm}$ in the transverse plane, and must occur at a point along the beam axis for which $|z|<18 \mathrm{~cm}$. In addition, the following criteria must be satisfied: (i) $p<p_{\text {beam }}+5 \times \sigma_{p}$, where $p$ and $p_{\text {beam }}$ are the track and incident beam momenta, respectively, and $\sigma_{p}$ is the momentum uncertainty for a charged track for which $p=p_{\text {beam }}$; (ii) $E<0.6 E_{\text {beam }}$, where $E$ is the BSC energy associated with the track, and $E_{\text {beam }}$ is the beam energy; (iii) $2<t<t_{p}+5 \times \sigma_{t}$ (in ns.), where $t$ is the measured time-of-flight for the track, $t_{p}$ is the time-of-flight calculated assigning the proton mass to the track, and $\sigma_{t}$ is the resolution of the barrel time-of-flight system.

After track selection, an event-level selection is imposed that requires the presence of at least two charged tracks, of which at least one satisfies all of the criteria listed above. In addition, the total energy deposited in the BSC $\left(E_{\text {sum }}\right)$ must be greater than $0.28 E_{\text {beam }}$, and the selected tracks must not all point into the forward $(\cos \theta>0)$ or the backward $(\cos \theta<0)$ hemisphere. 
For two-prong events, residual cosmic ray and lepton pair $\left(e^{+} e^{-}\right.$and $\left.\mu^{+} \mu^{-}\right)$backgrounds are removed by requiring that the tracks not be back-to-back, and that there be at least two isolated energy clusters in the BSC with $E>100 \mathrm{MeV}$ that are at least $15^{\circ}$ in azimuth from the closest charged track. This last requirement rejects radiative Bhabha events.

These requirements eliminate virtually all cosmic rays and most of the lepton pair $\left(e^{+} e^{-}\right.$and $\left.\mu^{+} \mu^{-}\right)$events. The remaining background contributions due to lepton pairs $\left(N_{l l}\right)$, including $\tau^{+} \tau^{-}$production above threshold, and two-photon events $\left(N_{\gamma \gamma}\right)$ are estimated using Monte Carlo simulations and subtracted as indicated in Eq.

The numbers of hadronic events and beam-associated background events are determined by fitting the distribution of event vertices along the beam direction with a Gaussian to describe the hadronic events and a polynomial of degree one to three for the beamassociated background. This background varies from of the selected hadronic event candidates, depending on the energy. The fit using a second degree polynomial, shown in Fig. IIi, , turned out to be the best. The difference between using a polynomial of degree one or three to that of degree two is about $1 \%$, which is included in the systematic error in the event selection.

The beam-associated background can also be determined by applying the same hadronic event selection criteria to separated-beam data, as described in ref. [3] 2$]$. The differences between the $R$ values obtained using these two methods range between 0.3 and $2.3 \%$, depending on the energy, and are included in the systematic uncertainty.

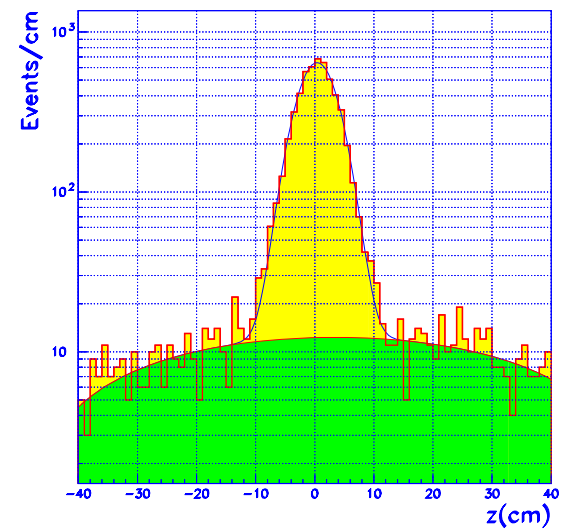

Figure 1: Event $V_{z}$ distribution at $\mathrm{Ecm}=2.6 \mathrm{GeV}$. fitted by a Gaussian plus a polynomial of degree 2. The Gaussian peak area represents the number of hadrons.

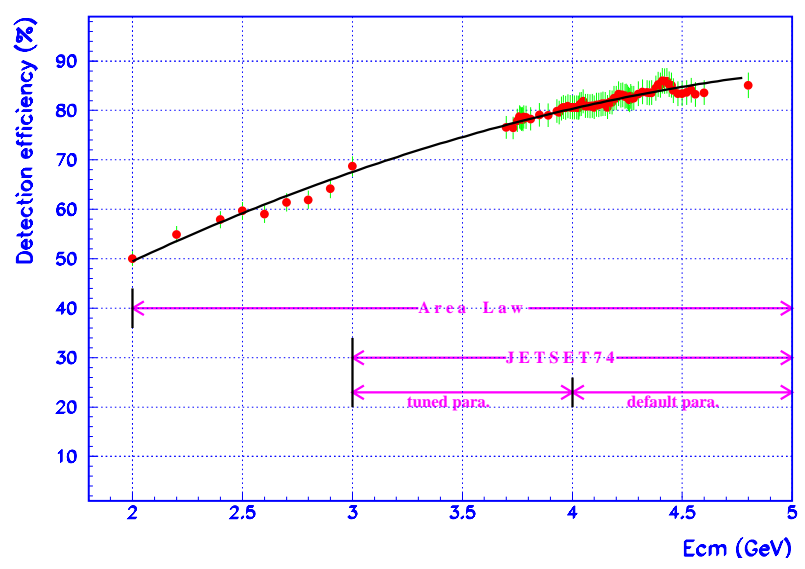

Figure 2: Detection efficiency versus energy in '99 $\mathrm{R}$ scan

The detection efficiencies were determined using JETSET74 for the energies above 3 $\mathrm{GeV}$.

$E_{c m}>4 \mathrm{GeV}$ with default parameters;

$E_{c m} \leq 4 \mathrm{GeV}$ with tuned parameters:

$$
\begin{aligned}
& a=0.3 \rightarrow 1.5, b=0.58 \rightarrow 0.78 \\
& w=0.8 \rightarrow 0.5, \sigma=0.36 \rightarrow 0.45 .
\end{aligned}
$$


A special joint effort was made by the Lund group and the BES collaboration to develop a new generator based on Lund Area-Law [i, $\left.\bar{i}_{-1}\right]$ to describe few-body states, which extremely necessary in low energy region $(\leq 3 \mathrm{GeV})$. The difference between the JETSET74 and LUARLW results is about 1\%, and is also taken into account in estimating the systematic uncertainty(see Table $12 \overline{2}$ ).

Above $3.77 \mathrm{GeV}$, the production of $D, D^{*}, D_{s}$, and $D_{s}^{*}$ is included in the generator according to the Eichten Model [8]⿱一⿱㇒⿵冂⿰丨丨寸].]. A A Monte Carlo event generator has been developed to handle decays of the resonances in the radiative return processes $e^{+} e^{-} \rightarrow \gamma J / \psi$ or

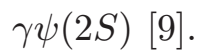

Figure ${ }_{2}^{2}$ shows the variation of the detection efficiency as a function of $\mathrm{cm}$ energy.

\section{Luminosity Measurement}

The integrated luminosity is determined from the number of large-angle Bhabha events, as described in detail in ref. [2] The luminosity systematic error includes the uncertainties arising in the selection of the Bhabha events, the efficiency determination, the background contamination, and the

\begin{tabular}{|c|c|c|c|}
\hline \hline$E_{c m}(\mathrm{GeV})$ & 3.0 & 4.6 & 4.8 \\
\hline$\varepsilon($ JETSET) & 0.6733 & .8628 & .8796 \\
\hline$\varepsilon$ (AreaLaw) & 0.6969 & .8757 & .8664 \\
\hline diff. (\%) & 3.5 & 1.5 & 1.5 \\
\hline \hline
\end{tabular}

Table 2: $\varepsilon$ comparison between AreaLaw and Jetset cross section calculation.

\section{Initial State Radiative Correction}

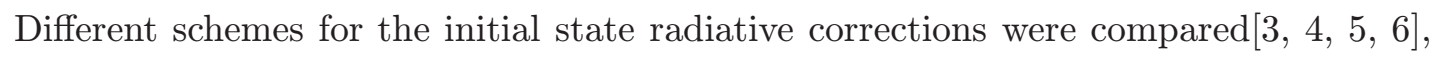
as reported in ref. [2i]. Below charm threshold, the four different schemes agree with each other to within $1 \%$, while above charm threshold, where resonances are important, the agreement is within 1 to $3 \%$. The radiative correction used in this analysis is based on ref. [i,i], and the differences with the other schemes are included in the systematic error.

\section{Final Results and Contributions}

Table $\bar{p}$ lists the values of $R$ from this experiment. They are displayed in Fig.

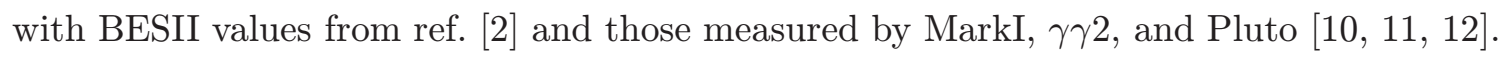
The $R$ values from BESII have an average uncertainty of about $6.6 \%$, which represents a factor of two to three improvement in precision in the 2 to $5 \mathrm{GeV}$ energy region. Of this error, $4.3 \%$ is common to all points. These improved measurements have a significant impact on the global fit to the electroweak data and the determination of the SM prediction

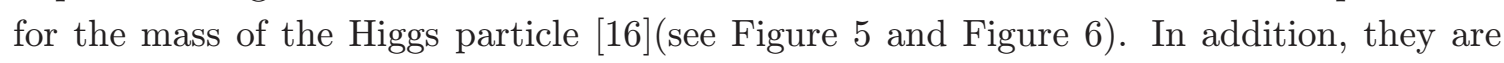
expected to provide an improvement in the precision of the calculated value of $a_{\mu}^{S M}$ and

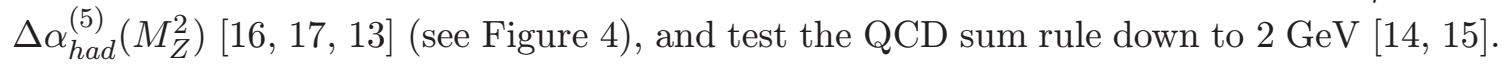

\section{References}

[1] J.Z. Bai et al., BES Collab., Nucl. Instr. Meth. A458 627-637(2001)

[2] J. Z. Bai et al., (BES Collab.) Phys. Rev. Lett. 84594 (2000).

[3] F.A. Berends and R. Kleiss, Nucl. Phys. B178 141 (1981). 
Table 3: Values of $\mathrm{R}$ from this experiment; the first error is statistical, the second systematic.

\begin{tabular}{|c|c|c|c|c|c|c|c|}
\hline $\begin{array}{c}E_{c m} \\
(\mathrm{GeV})\end{array}$ & $R$ & $\begin{array}{c}E_{c m} \\
(\mathrm{GeV})\end{array}$ & $R$ & $\begin{array}{c}E_{c m} \\
(\mathrm{GeV})\end{array}$ & $R$ & $\begin{array}{c}E_{c m} \\
(\mathrm{GeV})\end{array}$ & $R$ \\
\hline 2.000 & $2.18 \pm 0.07 \pm 0.18$ & 3.890 & $2.64 \pm 0.11 \pm 0.15$ & 4.120 & $4.11 \pm 0.24 \pm 0.23$ & 4.340 & $3.27 \pm 0.15 \pm 0.18$ \\
\hline 2.200 & $2.38 \pm 0.07 \pm 0.17$ & 3.930 & $3.18 \pm 0.14 \pm 0.17$ & 4.130 & $3.99 \pm 0.15 \pm 0.17$ & 4.350 & $3.49 \pm 0.14 \pm 0.14$ \\
\hline 2.400 & $2.38 \pm 0.07 \pm 0.14$ & 3.940 & $2.94 \pm 0.13 \pm 0.19$ & 4.140 & $3.83 \pm 0.15 \pm 0.18$ & 4.360 & $3.47 \pm 0.13 \pm 0.18$ \\
\hline 2.500 & $2.39 \pm 0.08 \pm 0.15$ & 3.950 & $2.97 \pm 0.13 \pm 0.17$ & 4.150 & $4.21 \pm 0.18 \pm 0.19$ & 4.380 & $3.50 \pm 0.15 \pm 0.17$ \\
\hline 2.600 & $2.38 \pm 0.06 \pm 0.15$ & 3.960 & $2.79 \pm 0.12 \pm 0.17$ & 4.160 & $4.12 \pm 0.15 \pm 0.16$ & 4.390 & $3.48 \pm 0.16 \pm 0.16$ \\
\hline 2.700 & $2.30 \pm 0.07 \pm 0.13$ & 3.970 & $3.29 \pm 0.13 \pm 0.13$ & 4.170 & $4.12 \pm 0.15 \pm 0.19$ & 4.400 & $3.91 \pm 0.16 \pm 0.19$ \\
\hline 2.800 & $2.17 \pm 0.06 \pm 0.14$ & 3.980 & $3.13 \pm 0.14 \pm 0.16$ & 4.180 & $4.18 \pm 0.17 \pm 0.18$ & 4.410 & $3.79 \pm 0.15 \pm 0.20$ \\
\hline 2.900 & $2.22 \pm 0.07 \pm 0.13$ & 3.990 & $3.06 \pm 0.15 \pm 0.18$ & 4.190 & $4.01 \pm 0.14 \pm 0.14$ & 4.420 & $3.68 \pm 0.14 \pm 0.17$ \\
\hline 3.000 & $2.21 \pm 0.05 \pm 0.11$ & 4.000 & $3.16 \pm 0.14 \pm 0.15$ & 4.200 & $3.87 \pm 0.16 \pm 0.16$ & 4.430 & $4.02 \pm 0.16 \pm 0.20$ \\
\hline 3.700 & $2.23 \pm 0.08 \pm 0.08$ & 4.010 & $3.53 \pm 0.16 \pm 0.20$ & 4.210 & $3.20 \pm 0.16 \pm 0.17$ & 4.440 & $3.85 \pm 0.17 \pm 0.17$ \\
\hline 3.730 & $2.10 \pm 0.08 \pm 0.14$ & 4.020 & $4.43 \pm 0.16 \pm 0.21$ & 4.220 & $3.62 \pm 0.15 \pm 0.20$ & 4.450 & $3.75 \pm 0.15 \pm 0.17$ \\
\hline 3.750 & $2.47 \pm 0.09 \pm 0.12$ & 4.027 & $4.58 \pm 0.18 \pm 0.21$ & 4.230 & $3.21 \pm 0.13 \pm 0.15$ & 4.460 & $3.66 \pm 0.17 \pm 0.16$ \\
\hline 3.760 & $2.77 \pm 0.11 \pm 0.13$ & 4.030 & $4.58 \pm 0.20 \pm 0.23$ & 4.240 & $3.24 \pm 0.12 \pm 0.15$ & 4.480 & $3.54 \pm 0.17 \pm 0.18$ \\
\hline 3.764 & $3.29 \pm 0.27 \pm 0.29$ & 4.033 & $4.32 \pm 0.17 \pm 0.22$ & 4.245 & $2.97 \pm 0.11 \pm 0.14$ & 4.500 & $3.49 \pm 0.14 \pm 0.15$ \\
\hline 3.768 & $3.80 \pm 0.33 \pm 0.25$ & 4.040 & $4.40 \pm 0.17 \pm 0.19$ & 4.250 & $2.71 \pm 0.12 \pm 0.13$ & 4.520 & $3.25 \pm 0.13 \pm 0.15$ \\
\hline 3.770 & $3.55 \pm 0.14 \pm 0.19$ & 4.050 & $4.23 \pm 0.17 \pm 0.22$ & 4.255 & $2.88 \pm 0.11 \pm 0.14$ & 4.540 & $3.23 \pm 0.14 \pm 0.18$ \\
\hline 3.772 & $3.12 \pm 0.24 \pm 0.23$ & 4.060 & $4.65 \pm 0.19 \pm 0.19$ & 4.260 & $2.97 \pm 0.11 \pm 0.14$ & 4.560 & $3.62 \pm 0.13 \pm 0.16$ \\
\hline 3.776 & $3.26 \pm 0.26 \pm 0.19$ & 4.070 & $4.14 \pm 0.20 \pm 0.19$ & 4.265 & $3.04 \pm 0.13 \pm 0.14$ & 4.600 & $3.31 \pm 0.11 \pm 0.16$ \\
\hline 3.780 & $3.28 \pm 0.12 \pm 0.12$ & 4.080 & $4.24 \pm 0.21 \pm 0.18$ & 4.270 & $3.26 \pm 0.12 \pm 0.16$ & 4.800 & $3.66 \pm 0.14 \pm 0.19$ \\
\hline 3.790 & $2.62 \pm 0.11 \pm 0.10$ & 4.090 & $4.06 \pm 0.17 \pm 0.18$ & 4.280 & $3.08 \pm 0.12 \pm 0.15$ & & \\
\hline 3.810 & $2.38 \pm 0.10 \pm 0.12$ & 4.100 & $3.97 \pm 0.16 \pm 0.18$ & 4.300 & $3.11 \pm 0.12 \pm 0.12$ & & \\
\hline 3.850 & $2.47 \pm 0.11 \pm 0.13$ & 4.110 & $3.92 \pm 0.16 \pm 0.19$ & 4.320 & $2.96 \pm 0.12 \pm 0.14$ & & \\
\hline
\end{tabular}

[4] G. Bonneau and F. Martin, Nucl. Phys. B27 387 (1971).

[5] E. A. Kuraev and V.S. Fadin, Sov. J. Nucl. Phys. 41, 3 (1985).

[6] A. Osterheld et al., SLAC-PUB-4160, 1986. (T/E)

[7] B. Andersson and Haiming Hu, "Few-body States in Lund String Fragmentation Model", hep-ph/9910285.

[8] E. Eichten et al., Phys. Rev. D21 203 (1980).

[9] J.C. Chen et al., Phys. Rev. D62 034003 (2000).

[10] J. L. Siegrist et al., (Mark I Collab.), Phys. Rev. D26 969(1982).

[11] C. Bacci et al., ( $\gamma \gamma 2$ Collab.), Phys. Lett. B86 234(1979).

[12] L. Criegee and G. Knies, (Pluto Collab.), Phys. Rep. 83151 (1982); Ch. Berger et al., Phys. Lett. B81 410(1979).

[13] A. Martin et al., Phys. Lett. B49269(2000).

[14] M. Davier and A. Hoecker, Phys. Lett. B419419(1998).

[15] J.H. Kuehn and M. Steinhauser, Phys. Lett. B437425(1998).

[16] H. Burkhardt and B. Pietrzyk, LAPP-EXP 2001-03,accepted by Physics Letters.

[17] B. Pietrzyk, Robert Carey, Atul Gurtu, talks at ICHEP2000, Osaka, Japan, July 2000.

[18] E. Tournefier, hep-ex/0105091. 

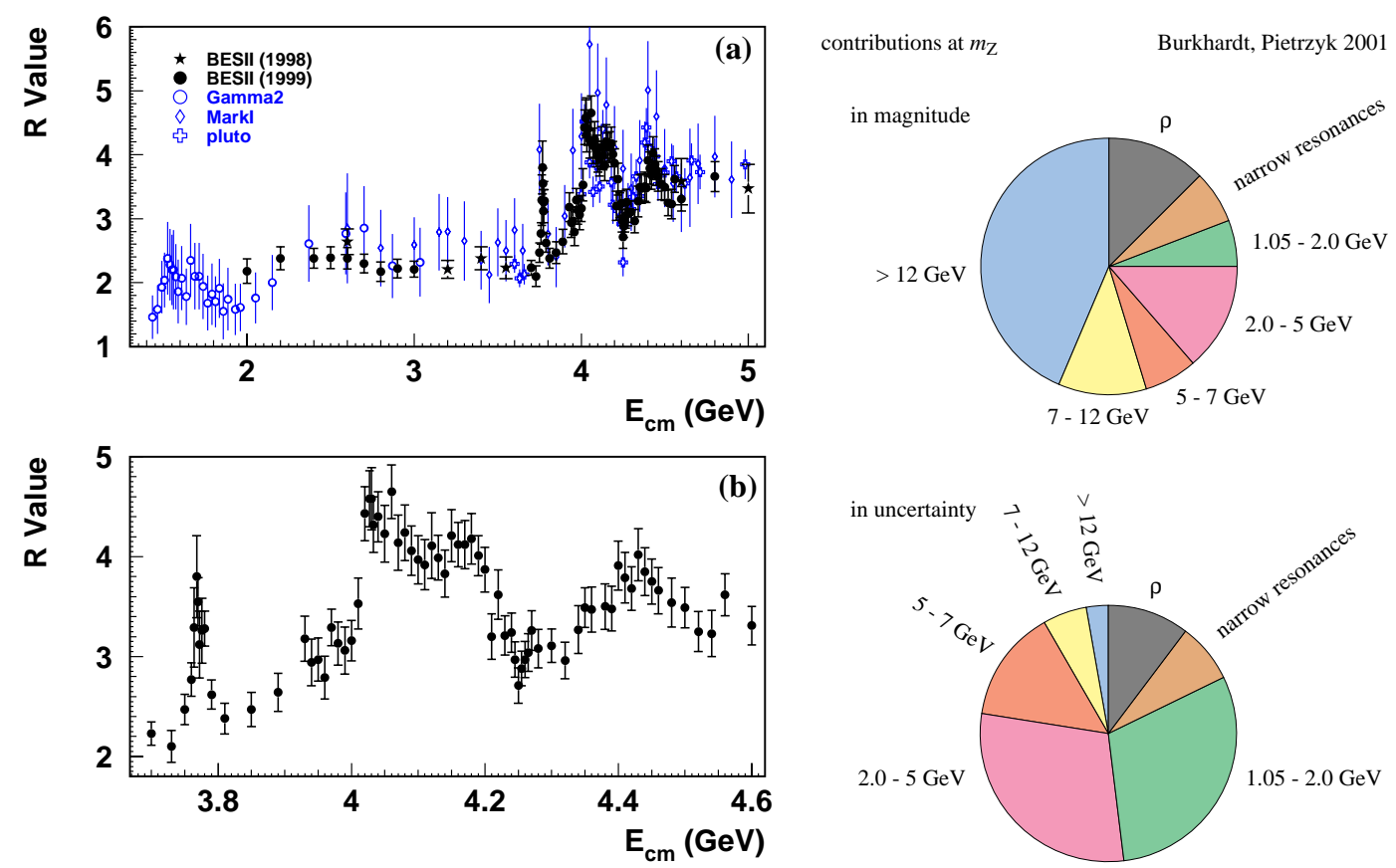

Figure 3: (a) A compilation of measurements of $R$ in the cm energy range from 1.4 to $5 \mathrm{GeV}$.

(b) $R$ values from this experiment in the resonance

Figure 4: Relative contributions to $\Delta \alpha_{\text {had }}^{(5)}\left(M_{Z}^{2}\right)$ in mangitude and uncertainty which including BES data contribution. region between 3.75 and $4.6 \mathrm{GeV}$.
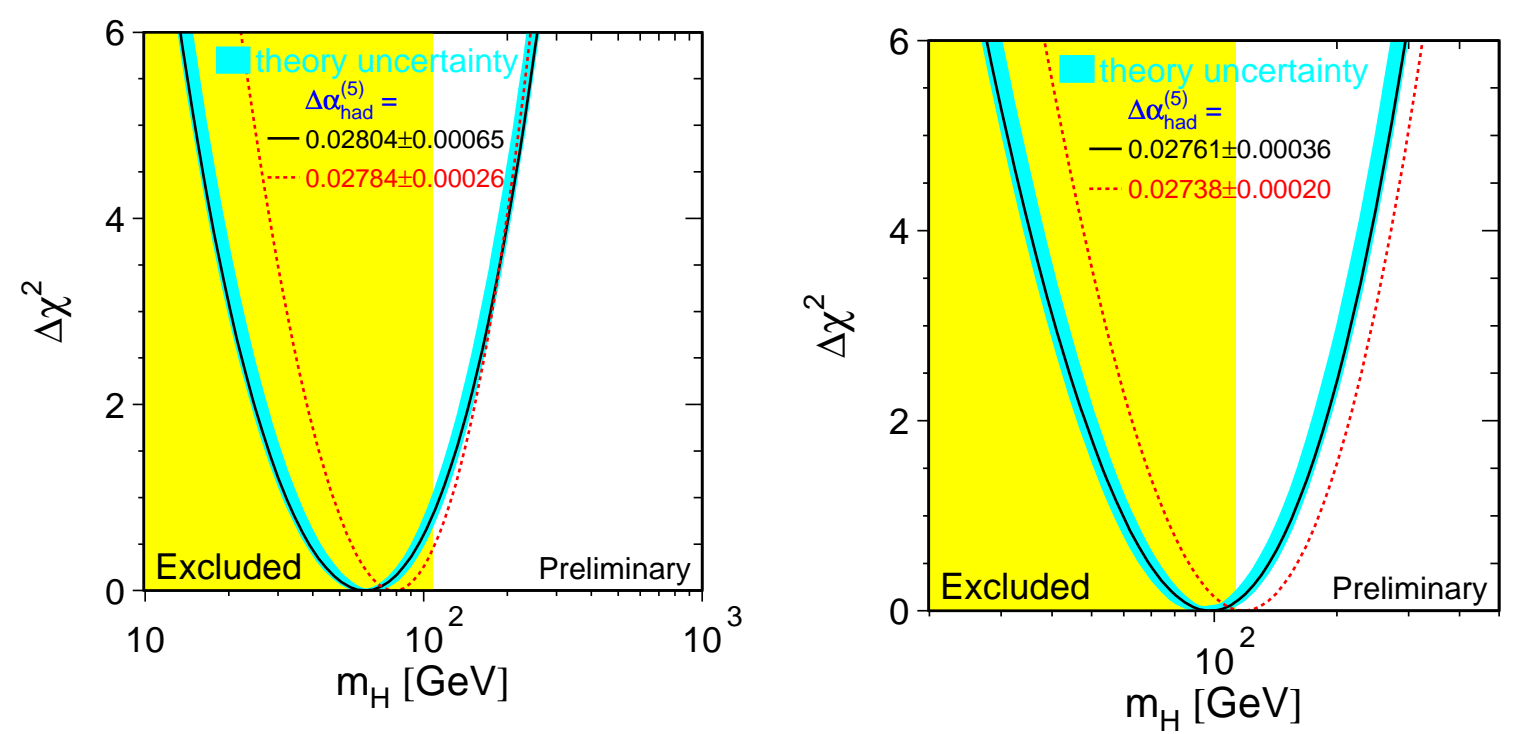

Figure 5: SM fit: $m_{H}=62_{-30}^{+53} \mathrm{GeV}$, and $m_{H}<170 \mathrm{GeV}$ at $95 \%$ C.L. (no BES) [1 1 i

Figure 6: SM fit: $m_{H}=98_{-38}^{+58} \mathrm{GeV}$, and $m_{H}<$ $212 \mathrm{GeV}$ at $95 \%$ C.L. (with BES) [1] 11,18 